\title{
Marine ecotoxicology
}

\author{
Christophe Minier • Philippe Garrigues
}

Published online: 11 December 2009

(C) Springer-Verlag 2009

The 15th International Symposium on Pollutant Responses in Marine Organisms (PRIMO15) was held in France, for the first time, on 17-20 May 2009. Bordeaux was the city chosen to host this international forum for exchanging state-of-the-art information focusing on xenobiotics, mechanisms of action, and relevance to aquatic ecosystems.

One special objective of this conference was to broaden our view in relation to new compounds, new areas, new mechanisms and new integrative concepts regarding xenobiotic effects in aquatic ecosystems. Accordingly, there were sessions on emerging compounds, including endocrinedisrupting compounds (EDCs), pharmaceuticals and personal care products (PPCPs), and nanoparticles. Notably, it was the first time that the two latter types of compounds had been discussed in sessions devoted to them during a PRIMO meeting.

Progress in analytical chemistry is crucial to the study of emerging compounds, and this is also the first time that Analytical and Bioanalytical Chemistry has

\footnotetext{
C. Minier

Laboratory of Ecotoxicology, University of Le Havre,

25, rue Philippe Lebon,

76058 Le Havre, France

e-mail: minier@univ-lehavre.fr

P. Garrigues $(\square)$

Institut des Sciences Moléculaires (ISM), UMR 5255 CNRS,

Université de Bordeaux 1,

33405 Talence Cedex, France

e-mail: p.garrigues@ism.u-bordeaux1.fr
}

included a collection of original articles presented during a PRIMO meeting. These contributions exemplify some of the key points that were discussed in the sessions. They include presentations on the occurrence of emerging compounds in aquatic systems, effects on nontarget organisms, and explorations of the mechanisms involved.

Some endocrine and pharmaceutically active compounds have been known for more than two decades to enter the environment. Nevertheless, a larger picture has emerged recently because of continuous improvements in chemical analysis methodologies, which have enabled the limits of detection to be lowered for a wide array of xenobiotics in environmental matrices. Compounds such as pharmaceuticals can now be detected in marine ecosystems, and may be found at close to toxicologically relevant concentrations in freshwater. The full extent and magnitude of the presence of these EDCs and pharmaceuticals in the aquatic environment are largely unknown, but presentations and discussions during PRIMO15 showed that a number of effects on aquatic life can arise from these compounds. It is well known that pharmaceuticals, EDCs and nanoparticles display complex responses and exert their actions upon multiple targets. Variety and complexity is decupled when studying aquatic organisms, as both expected (based on mammalian toxicology) and unexpected effects can be observed on nontarget organisms. Exploring and understanding the underlying mechanisms of toxicity is one of the major goals of ecotoxicological studies. Recent advances in our knowledge of the metabolism of emerging compounds and their interactions with receptors, for example, were presented. Omics have also been deployed, 
and they have shown that a wide array of effects and mechanisms are yet to be elucided.

The conference was attended by more than 300 scientists, including 100 young researchers (Ph.D. students and postdocs) from around 30 countries. The scientific program included a plenary lecture given by Rudolf $\mathrm{Wu}$ (Hong Kong City University), 80 oral presentations, and 10 young researcher flash presentations. Furthermore, 250 posters were presented during the conference. Seven awards were attributed to specific young researcher presentations: Jamius W.Y. Yeung (University of Hong Kong, P.R. China), Susan Bengtson Nash (University of Queensland, Australia), Stephan Fischer (UFZ, Helmholtz Centre for Environmental Research, Germany), Tim Bean (Cefas Weymouth Laboratory, United Kingdom), Marta Popovic (Laboratory for Molecular Ecotoxicology, Zagreb, Croatia), Leena Mol Thuruthippallil (Center for Marine Environmental Studies, Matsuyama, Japan), and Nathalie Hinfray (INERIS, France).

The pleasant venue of the meeting - the Bordeaux Conference Centre - and the enjoyable social events held during it (Sunday night get-together party, conference dinner in a wine chateau with more than 100 different Bordeaux wines served, great buffet lunches in the French classical tradition) complemented the symposium.

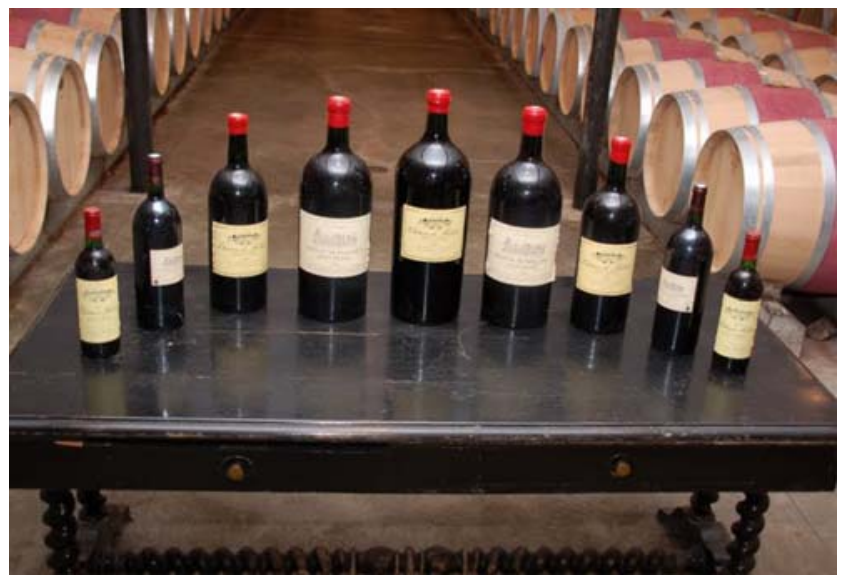

The program for PRIMO15, abstracts and more are still available at: http://primo15.ism.u-bordeaux1.fr/.

PRIMO16 will take place in Long Beach Island, California, on 15-18 May 2011.

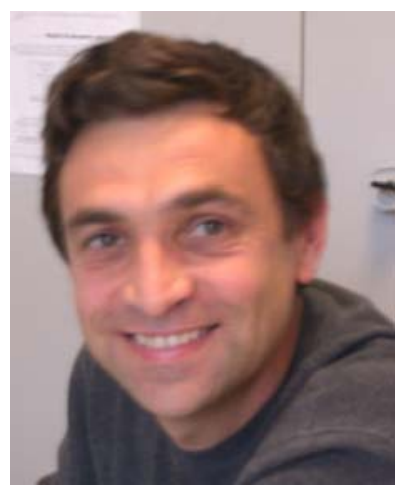

Christophe Minier is professor at the University of Le Havre and head of the Etiology of Environmental Stresses group within the Laboratory of Ecotoxicology (LEMA). He is the coordinator of research activities on endocrine disruption for the national consortium "EXECO" (a group of 6 French laboratories), and the leader of the European research program Risk Analysis Associated with Endocrine DisruptionRAED since 2002. Dr Minier's research activities are in the area of endocrine disruption, environmental genotoxicity and studies on the multixenobiotic resistance (MXR) system. His particular focus is on the assessment of exposure (bioavailability and interactions with $\mathrm{ABC}$ transporters), and its effects at several levels of biological organization (from molecular components to the whole organism). The main organisms he studies are fish and mollusks living in both marine and freshwater environments.

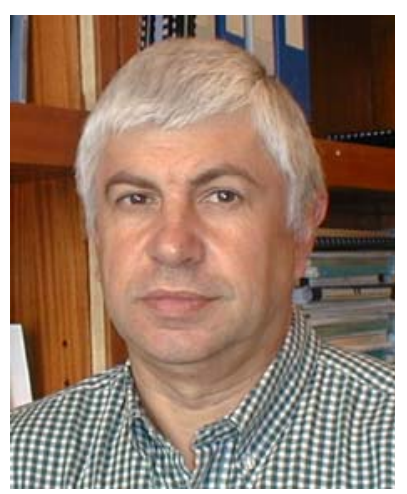

Philippe Garrigues is a CNRS research director and is currently the head of the Institute of Molecular Sciences (ISM, UMR 5255 CNRS) at the University of Bordeaux. His research interests are the analytical aspects (chromatographies and spectroscopies) related to the detection of organic pollutants and their environmental fate and toxicological effects. $\mathrm{He}$ has been involved in the development of biochemical markers as early warning systems for the healh assessment of marine ecosystems through the coordination of large research projects (i.e., GICBEM, BIOMAR, BEEP) supported by the DG Research (European Commission, Brussels). Dr. Garrigues has authored about 180 publications. He was a member of the executive committee of SETAC Europe (97-99) and chairman of the division "Chemistry in the Environment" (DCE) of the European Association for Chemical and Molecular Sciences ( EuCheMS, 03-08). He has also organized various congresses, most of which have been related to international scientific societies (SETAC, ISPAC, EuCheMS). 